\title{
Conhecimento atual sobre os tentaculitoideos devonianos das bacias do Amazonas e Parnaíba, Brasil, depositados em instituições brasileiras Current knowledge about Devonian tentaculitoids of Amazonas and Parnaíba basins, Brazil, deposited in Brazilian institutions
}

\author{
Jeanninny Carla Comniskey', Renato Pirani Ghilardi", Elvio Pinto BosettillI \\ Universidade de São Paulo. Ribeirão Preto, São Paulo, Brasil \\ "Universidade Estadual Paulista "Júlio de Mesquita Filho". Bauru, São Paulo, Brasil \\ I'U Universidade Estadual de Ponta Grossa. Ponta Grossa, Paraná, Brasil
}

\begin{abstract}
Resumo: Os tentaculitoideos são encontrados em afloramentos do Devoniano das bacias do Paraná (Formações Ponta Grossa e São Domingos), Amazonas (Formações Maecuru e Ererê) e Parnaíba (Formação Cabeças). Foram analisadas 39 amostras de coleções científicas provenientes do Devoniano Médio das bacias do Amazonas e do Parnaíba, com principal objetivo de realizar uma revisão sistemática e refinar o conhecimento acerca da distribuição estratigráfica e paleogeográfica do grupo. As espécies encontradas foram: Tentaculites trombetensis, Tentaculites stubeli, Tentaculites eldredgianus, Tentaculites oseryi e Styliolina clavulus. As espécies T. eldredgianus, T. trombetensis e S. clavulus foram encontradas em afloramentos do Eifeliano até o Givetiano, enquanto T. oseryi apenas no Givetiano e T. stubeli no Eifeliano. As primeiras ocorrências de tentaculitoideos para a América do Sul são registradas no Siluriano, o que corrobora a hipótese de que durante o Siluriano Inferior ocorreu um grande aumento da diversidade e quantidade de tentaculitoideos no mundo.
\end{abstract}

Palavras-chave: Tentaculitoidea. Sistemática. Devoniano. Paleobiogeografia. Bacia do Amazonas. Bacia do Parnaíba.

\begin{abstract}
The Tentaculitoids are commonly found in the outcrops of the Devonian of the Paraná Basin (Ponta Grossa and São Domingos Formation), the Amazon Basin (Maecuru and Ererê Formation) and Parnaíba Basin (Cabeças Formation). We analyzed 39 samples of scientific collections from the middle Devonian of the Amazon and Parnaíba Basin, with main objective to conduct a systematic review to refine the knowledge on the stratigraphic and paleogeographic distribution of the group. The species found were: Tentaculites trombetensis, Tentaculites stubeli, Tentaculites eldredgianus, Tentaculites oseryi and Styliolina clavulus. The species T. eldredgianus, T. trombetensis and S. clavulus were found in outcrops of Eifelian to the Givetian. While T. oseryi only during Givetian and T. stubeli during Eifelian. The first occurrences of tentaculitoids in South America are related to the Silurian, which corroborates the hypothesis that during the Early Silurian, there was a large increase in the diversity and quantity of tentaculitoids in the world.
\end{abstract}

Keywords: Tentaculitoidea. Systematic. Devonian. Palaeobiogeography. Amazonas Basin. Parnaíba Basin.

COMNISKEY, J. C., R. P. GHILARDI \& E. P. BOSETTI, 2015. Conhecimento atual sobre os tentaculitoideos devonianos das bacias do Amazonas e Parnaíba, Brasil, depositados em instituições brasileiras. Boletim do Museu Paraense Emílio Goeldi. Ciências Naturais 10(1): 49-61. Autor para correspondência: Jeanninny Carla Comniskey. Universidade de São Paulo. Pós-Graduação em Biologia Comparada. Av. Bandeirantes, 3900 - Monte Alegre. Ribeirão Preto, SP, Brasil. CEP 14040-901 (comniskey@gmail.com).

Recebido em 18/11/2014

Aprovado em 14/05/2015

Responsabilidade editorial: Hilton Tulio Costi

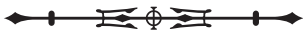




\section{INTRODUÇÃO}

O estudo da classe Tentaculitoidea tem avançado muito no decorrer dos anos, porém pouco se sabe sobre o animal que habita a concha ou em qual reino animal o táxon deve ser incluído. Segundo Wei et al. (2012), existem duas principais ideias quanto à posição taxonômica do grupo. A primeira seria uma afinidade com os moluscos devido à semelhança com a concha e as paredes dela (Bouček, 1964; Blind, 1969; Lardeux, 1969; Farsan, 1994; Schindler, 2012). Já a segunda linha de pesquisas reconhece os tentaculitoideos como um grupo irmão dos microconchídeos (Weedon, 1991; Vinn \& Taylor, 2007; Zatoń \& Taylor, 2009; Vinn, 2010; Vinn \& Zatoń, 2012; Vinn, 2013). Embora estas duas hipóteses ainda sejam muito discutidas, prefere-se adotar, neste trabalho, a primeira hipótese taxonômica para este grupo.
Dessa forma, os tentaculitoideos são invertebrados extintos que possuem concha carbonática coniforme de tamanho milimétrico. A maioria apresenta a concha reta, sendo que poucos apresentam concha encurvada e até mesmo enrolada (Schindler, 2012). Podem possuir uma concha lisa ou com ornamentação e, neste último caso, apresentam anéis, microanéis, macroanéis, estrias e espaços interanulares (Figura 1). A classificação dos tentaculitoideos é baseada, exclusivamente, em aspectos morfológicos da concha, a partir da ornamentação externa (Ciguel, 1989; Azevedo-Soares, 1999; Comniskey \& Ghilardi, 2013). A classe Tentaculitoidea Lyashenko, 1957 (= Cricoconarida Fisher, 1962) é dividida em três ordens: Tentaculitida Ljashenko, 1955, Homoctenida Bouček, 1964 e Dacryoconarida Fisher, 1962.

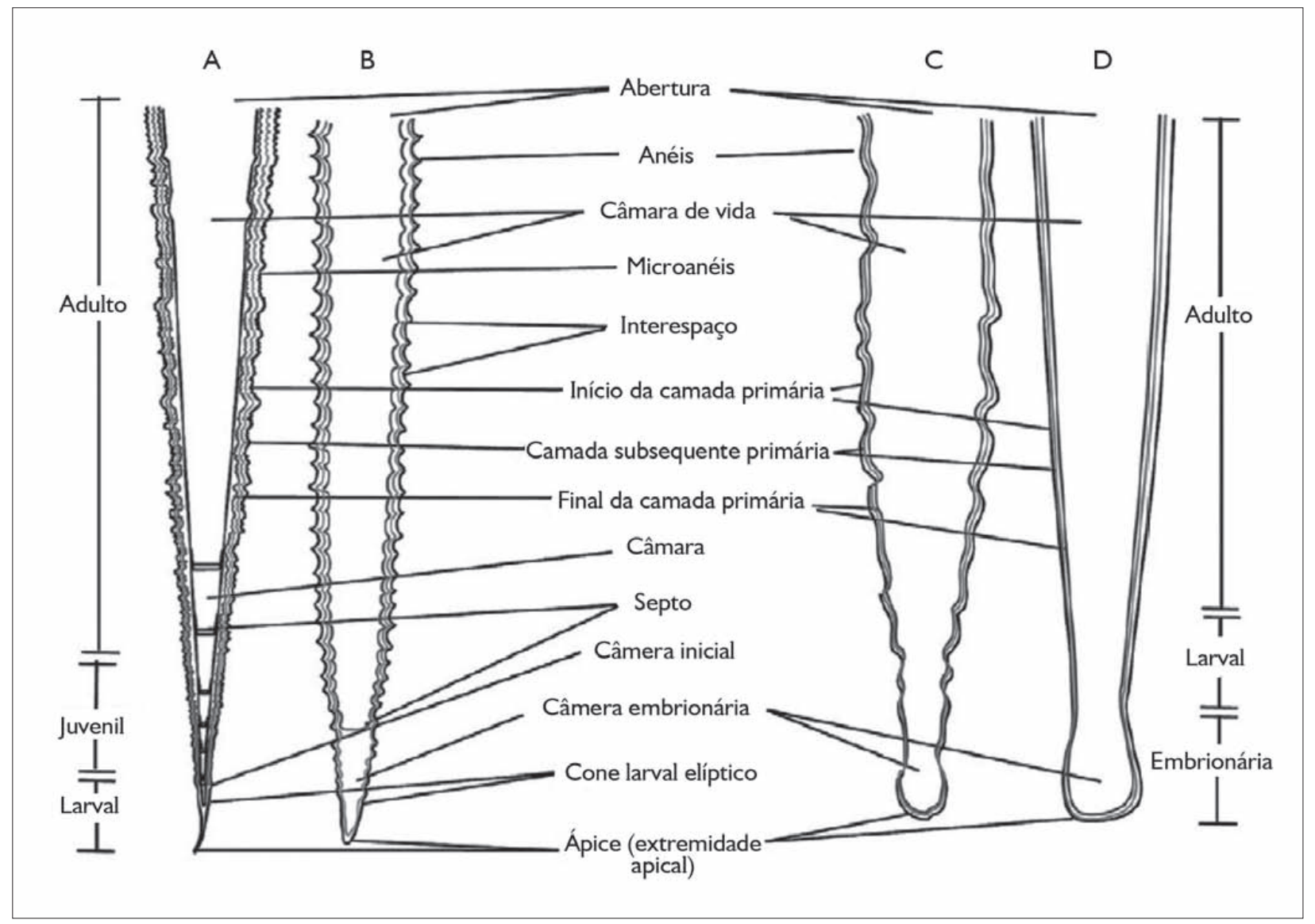

Figura 1. Representantes das ordens Tentaculitida (A), Homoctenida? (B) e Dacryoconarida? (C e D). Modificado de Wittmer \& Miller (2011).

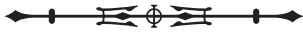


Segundo Fisher \& Young (1955), os primeiros registros de tentaculitídeos são do Ordoviciano Inferior da Formação Chepultepec, da Virginia (Tremadociano), com representantes da ordem Tentaculitida ocorrendo em abundância e ficando documentados no registro fossilífero até o término do Siluriano. O táxon dos dacryoconarideos surge no Siluriano, mas torna-se excepcionalmente diverso no Devoniano Inferior e Médio (Schindler, 2012). Já os homoctenídeos têm seu registro iniciado a partir do Devoniano e seu ápice evolutivo ocorre no Frasniano (Bond, 2006; Schindler, 2012). Este período é caracterizado pela grande expansão da diversidade e da distribuição geográfica dos tentaculitoideos (Wittmer \& Miller, 2011; Schindler, 2012). Schindler (1990, 2012) e Bond (2006) afirmam que os homoctenideos foram os únicos tentaculitoideos que ultrapassaram a barreira da extinção Frasniano - Fameniano (FF).

No Brasil, os tentaculitoideos são encontrados nas bacias do Paraná, Amazonas e Parnaíba. Na Bacia do Paraná, foi registrada a maior descrição de gêneros Tentaculites, Homoctenus, Uniconus, Multiconus, Dicricoconus, Volynites e Styliolina, enquanto que nas bacias do Amazonas e do Parnaíba apenas os gêneros Tentaculites e Styliolina são registrados. Apesar de ser um grupo cosmopolita no Devoniano brasileiro, há poucos trabalhos que lidam com a sistemática do grupo. Os trabalhos de Hartt \& Rathbun (1875), Clarke (1899a, 1899b) e Katzer (1903) foram os pioneiros na descrição de espécies do grupo para o Siluriano e Devoniano. Porém, após as primeiras descrições de espécies, poucos são os trabalhos de caráter taxonômico desses táxons e, conforme indicado em Comniskey \& Ghilardi (dados não publicados), os realizados não seguem os critérios relacionados no Código Internacional de Nomenclatura Zoológica, tornado-os, em sua maioria, inválidos (ver Ciguel et al., 1984; Ciguel \& Rosler, 1985; Ciguel et al., 1987; Ciguel, 1989; Azevedo-Soares, 1999, para exemplos na Bacia do Paraná). Nas bacias do Amazonas e Parnaíba, os trabalhos atuais relatam apenas a ocorrência dos tentaculitoideos, sem tratar especificamente sobre sua sistemática (Melo, 1985; Fonseca, 2004; Ponciano \& Machado, 2007; Ponciano, 2011; Ponciano et al., 2012).

Levando em consideração a falta de trabalhos sobre a sistemática do grupo, realizou-se uma revisão para as bacias do Amazonas e do Parnaíba, indicando as ocorrências das espécies ou formas aparentadas em outras localidades no mundo. Através da revisão realizada, foi possível correlacionar as ocorrências dos táxons encontrados entre as bacias sedimentares paleozoicas brasileiras e na região sul-americana, bem como atualizar e ampliar as informações paleobiogeográficas que esses táxons possam trazer.

\section{MATERIAL E MÉTODOS}

Foram realizadas visitas a coleções técnicas de universidades, museus e instituições de pesquisa. As coleções analisadas foram: Núcleo de Estudos Paleontológicos da Universidade Estadual do Rio de Janeiro (UERJ), Laboratório de Paleontologia de Invertebrados do Museu Nacional, Universidade Federal do Rio de Janeiro (MN/UFRJ), Departamento Nacional de Produção Mineral (DNPM) e Laboratório de Estudos de Comunidades Paleozoicas, da Universidade Federal do Estado do Rio de Janeiro (UNIRIO). Foram escolhidas estas coleções científicas em razão de os autores terem realizado contato com os seus curadores e, entre elas, foi verificada a presença dos holótipos do material dos trabalhos de Clarke (1899a, 1899b) e uma quantidade razoável de fósseis de tentaculitoideos. Não foi possível localizar os exemplares do trabalho de Hartt \& Rathbun (1875), nem consultando o banco de dados de fósseis de invertebrados catalogados no museu Paleontological Research Institution da Universidade de Cornell (Ithaca, Estados Unidos), nem por meio do contato com os responsáveis pela coleção.

Foram observadas 39 amostras, com aproximadamente 153 espécimes (34 da Bacia do Amazonas e 119 da Bacia do Parnaíba). O material analisado é proveniente de afloramentos do Devoniano Médio das Formações Maecuru e Ererê (Bacia do Amazonas) e da Formação 
Cabeças (Bacia do Parnaíba). Em algumas amostras, não foi possível chegar ao nível genérico ou específico, pela falta de caracteres morfológicos ou má preservação dos espécimes. Ressalta-se aqui que a maioria dos exemplares analisados foi coletada durante expedições do século passado, sem a preocupação com um registro estratigráfico mais detalhado. Todo o material analisado encontra-se depositado nas instituições de pesquisas visitadas (Figura 2).
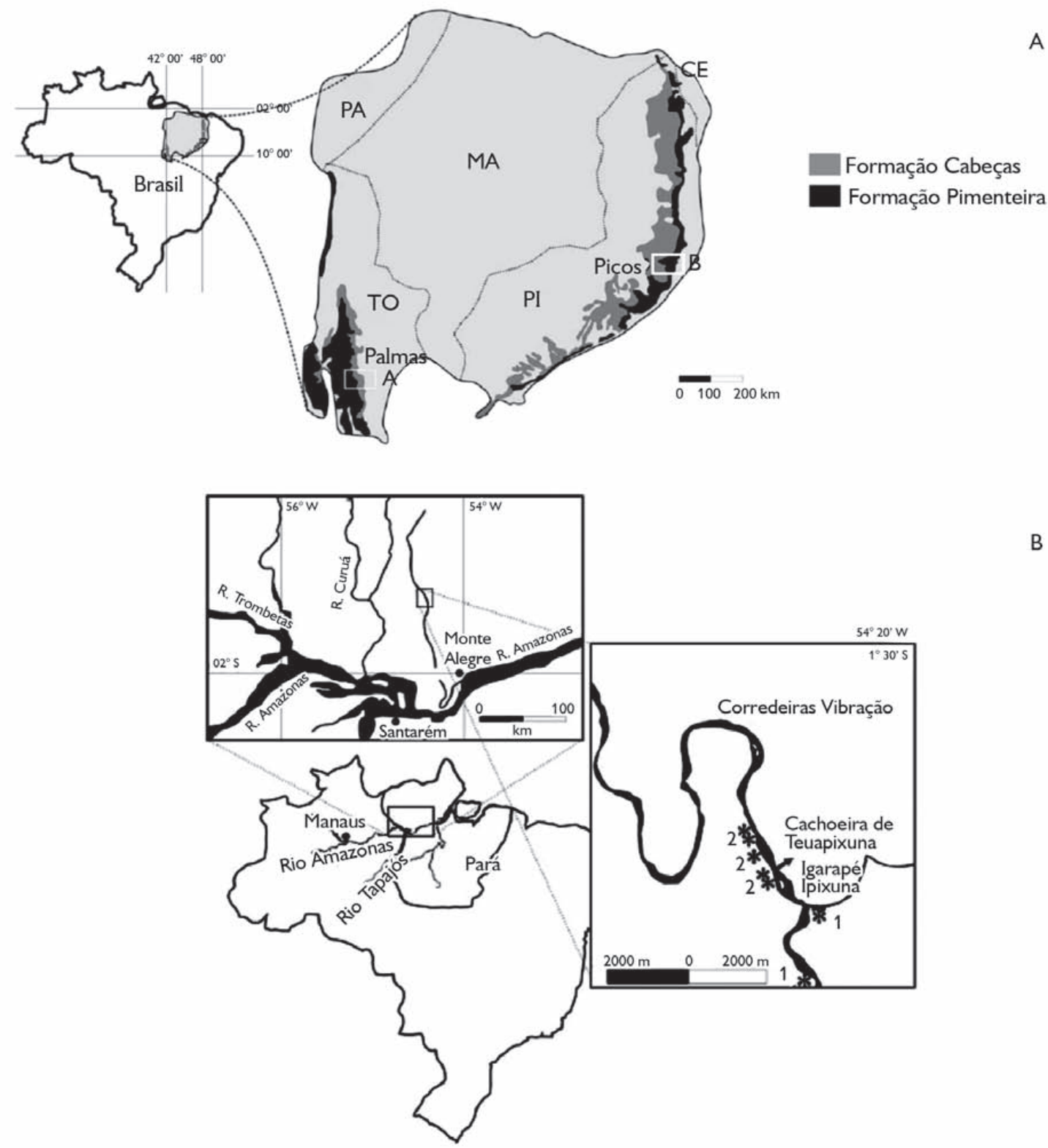

Figura 2. Localização dos afloramentos: A) Bacia do Parnaíba, localização das Formações Cabeças e Pimenteiras; B) Bacia do Amazonas, o ponto de número 1 refere-se aos afloramentos da Formação Ererê e os pontos de número 2 correspondem aos afloramentos da Formação Maecuru (modificado de Scheffler, 2010).

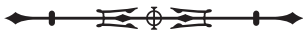




\section{SISTEMÁTICA PALEONTOLÓGICA}

Classe Tentaculitoidea Lyashenko, 1957

Ordem Tentaculitida Lyashenko, 1955

Família Tentaculitidae Walcott, 1886

Gênero Tentaculites Schloteim, 1820

Espécie-tipo Tentaculites scalaris Schlotheim, 1820

Tentaculites eldredgianus Hartt \& Rathbun, 1875 (Figura 3D)

1899 - Tentaculites eldredgianus Clarke, 1899, p. 80-81, plate IV, figuras 29-31

1933 - Tentaculites eldredgianus Katzer, 1933, p. 252, plate XIII, figuras 25-26

Diagnose específica:

Shell small, rather long, straight, circular in crosssection, at least $1 \mathrm{~mm}$ in diameter at the larger end, and tapering very gradually to an acute point. Length of the most perfect specimen, a fragment, about $16 \mathrm{~mm}$. Annulations narrow, quite prominent, an angular or slightly rounded of the summit; the interspaces are generally about twice as wide as the annulations, though they vary somewhat in width, and are flattened or a little rounded in the bottom; they are ornamented by fine annular raised lines, of which there are about four or five in each interspace, near the larger end of the specimen. The annulations decrease in size, but become more numerous toward the apex. There are about 5 to 7 in the space of $3 \mathrm{~mm}$ near the larger end (Hartt \& Rathbun, 1875).

Descrição: com tamanho aproximado de $1,8 \mathrm{~cm}$. Anéis arredondados, bastante proeminentes. Interespaços maiores na região adulta e da abertura, sendo aproximadamente duas vezes maiores do que os anéis. Anelamento irregular, podendo ocorrer áreas com pequeno espaçamento entre os anéis ou espaços com quase o tamanho dos anéis, também podendo ocorrer grandes áreas sem anéis. Câmara embrionária levemente arredondada.

Material: amostras de número 41 e 55, depositadas na Universidade Federal do Estado do Rio de Janeiro (UNIRIO).
Distribuição geográfica: A) rio Maecuru, margem direita, 400 m à montante da cachoeira Teuapixuna ou Alagação, coletadas pela expedição Orville Adelbert Derby, em 1986; B) localizado no lado norte de uma curva da BR-316 (Picos - Teresina), a cerca de 5 km do trevo de Picos, em direção a Oeiras, dentro de uma propriedade particular, no município de Picos (Deusana Machado, comunicação pessoal, 2014).

Distribuição estratigráfica: Formação Maecuru (Eifeliano) e Formação Cabeças (Givetiano).

Holótipo: o depósito do material descrito por Hartt \& Rathbun (1875), seja de seu holótipo, parátipos ou síntipos, não possui registro. Apesar de provavelmente estarem depositados na Cornell University, Estados Unidos, contatos realizados não foram suficientes para localizá-los. Dessa forma, é necessário, para validação da espécie, determinar seu neótipo.

Tentaculites stubeli Clarke, 1899 (Figura 3B)

Diagnose específica:

Concha um tanto robusta, estreitando-se rapidamente. A parte superior é cruzada por anneis mais ou menos oblíquos, muitas vezes irregulares, e separados por intervallos um tanto desiguaes. $\mathrm{Na}$ superfície externa estes anneis apresentam uma encosta superior comprida e uma costa inferior vertical ou mesmo côncava; nos moldes internos elles parecem agudamente angulares, com encostas iguaes em ambas as direções. Os intervallos são lisos e tornam-se mais estreitos para baixo, desapparecendo totalmente os anneis a uma distancia considerável do ápice. A parte apical da concha, na distancia de um terço a um quarto do comprimento total do tubo, é lisa (Clarke, 1899a).

Descrição: concha de tamanho médio de $2 \mathrm{~cm}$. Esta foi a maior espécie entre as analisadas. Anéis levemente oblíquos na região da abertura e interespaços irregulares. Anéis arredondados. Interespaços mais lisos e mais largos na região adulta e de abertura. Região apical lisa.

Material: amostras de número 5, 46 e 48 que estão depositadas na Universidade Federal do Estado do Rio de Janeiro (UNIRIO). 
Distribuição geográfica: encontrada no afloramento da Margem direita do rio Maecuru cerca de 400 m à montante da cachoeira de Teapuxina ou Alagação e cerca de 1,5 km à montante da foz do igarapé Ipixuna.

Distribuição estratigráfica: Formação Ererê (Neoeifeliana - Eogivetiana).

Holótipo: o material analisado por Clarke (1899a), seja de seu holótipo, parátipos ou síntipos, não possui registro. As figuras 24-28, estampa IV, do trabalho de Clarke (1899a) ficam designadas como síntipos. Apesar de provavelmente estarem depositados no Museu Nacional do Rio de Janeiro, contatos realizados não foram suficientes para localizá-los. Dessa forma, é necessário, para validação da espécie, determinar seu neótipo.

\section{Tentaculites oseryi Clarke, 1899 (Figura 3E)}

Diagnose específica:

\begin{abstract}
Esta espécie é menor do que T. stubeli ou T. trombetensis, e tem a superfície coberta de anneis regulares apertados, que são arredondados e separados por intervallos muito estreitos do que os anneis. Em algumas amostras os anneis se estendem quase até o ápice, em outras há um espaço considerável junto ao ápice que é liso. Os intervallos entre os anneis são fortemente côncavos e lisos. A maior amostra observada tem $9 \mathrm{~mm}$ de comprimento, e apresenta 23 anneis que se tornam rapidamente mais finos para baixo (Clarke, 1899a).
\end{abstract}

Descrição: anéis praticamente do tamanho dos espaços. Anéis arredondados, levemente espessos. Em alguns casos, região apical lisa. Interespaços fortemente côncavos e lisos. Em algumas amostras, a presença de anéis estende-se até o ápice, em outros casos ele está sem ornamentação. Tamanho aproximado de 1,3 cm. Clarke (1899a) afirma que esta espécie é menor do que T. trombetensis, porém, nas amostras analisadas, verificou-se o contrário.

Material: amostras de número 54, 58, 59 e 101, depositadas na Universidade Federal do Estado do Rio de Janeiro (UNIRIO).
Distribuição geográfica: A) afloramento localizado no lado norte de uma curva da BR-316 (Picos - Teresina), a cerca de $5 \mathrm{~km}$ do trevo de Picos em direção a Oeiras, dentro de uma propriedade particular, no município de Picos; B) situado em uma curva da estrada de terra em direção ao povoado de Oiti, a 800 m do entroncamento desta com a PI-120 (trecho Valença do Piauí - Pimenteiras), no município de Pimenteiras, Piauí (Deusana Machado, comunicação pessoal, 2014).

Distribuição estratigráfica: Formação Cabeças (Givetiano).

Holótipo: o material descrito por Clarke (1899a), seja de seu holótipo, parátipos ou sintipos, não possui registro. Apesar de provavelmente estarem depositados no Museu Nacional do Rio de Janeiro, contatos realizados não foram suficientes para localizá-los. Dessa forma, é necessário, para validação da espécie, determinar seu neótipo.

Tentaculites trombetensis Clarke, 1899 (Figura 3A)

1989 - Tentaculites trombetensis Ciguel, 1989, p. 64- 66, plates 24 e 25

Diagnose específica:

É uma concha pequena, recta, nunca flexuosa, e estreitando-se muito gradualmente. A superfície é coberta com annulações fortes e simples, cujos lados se inclinam de modo igualmente abrupto por cima e por baixo, e são separados por sulcos um tanto largos e fundos. Tanto as annullações como os sulcos se tornam mais estreitos e menores à medida que se approximam do ápice, e uma particularidade especial desta espécie é o forte desenvolvimento destas feições desde o começo da concha. Os sulcos intersticiaes são destituídos de ornamentação. O comprimento de uma amostra de tamanho médio, e provavelmente inteira, é de $11 \mathrm{~mm}$; a largura na abertura é de $1 \mathrm{~mm}$ (Clarke, 1899b).

Descrição: concha pequena, com tamanho aproximado de $0,8 \mathrm{~cm}$. Anéis arredondados. Interespaços largos e fundos. Anéis e espaços maiores na região da abertura. Ápice geralmente liso. Câmara embrionária levemente arredondada. 
Distribuição geográfica: A) rio Maecuru, margem direita a $400 \mathrm{~m}$ à montante da cachoeira Teuapixuna ou Alagação, coletadas pela Expedição Orvile A. Derby; B) localizado no lado norte de uma curva da BR-316 (Picos - Teresina), a cerca de $5 \mathrm{~km}$ do trevo de Picos, em direção a Oeiras, dentro de uma propriedade particular, no município de Picos (Deusana Machado, comunicação pessoal, 2014).

Distribuição estratigráfica: Formação Maecuru (Eifeliano) e Formação Cabeças (Givetiano).

Holótipo: o material analisado por Clarke (1899b), seja de seu holótipo, parátipos ou síntipos, não possui registro. As figuras 26-27, estampa II, do trabalho de Clarke (1899b) ficam designadas como síntipos. Apesar de provavelmente estarem depositados no Museu Nacional do Rio de Janeiro, contatos realizados não foram suficientes para localizá-los. Dessa forma, é necessário, para validação da espécie, determinar seu neótipo.

Classe Tentaculitoidea Lyashenko, 1957

Ordem Dacryoconarida Fisher, 1962

Família Stylionidae Grabau \& Shimer, 1910

Gênero Styliolina sp. Karpinsky, 1884

Espécie-tipo Styliola nucleata Karpinsky, 1884

Styliolina clavulus Barrande, 1852 (Figura 3C)

Diagnose específica:

La plupart des spécimens de cette espèce sont écrasés dans les roches schisteuses de nos bandes g 2-h 1. Cependant, on en découvre quelques uns qui permettent de reconnaitre la forme naturelle, conique et régulière de la coquille, dont la section transverse semble circulaire. La surface de ce test n'a conservé aucune trace d'ornemens. Dimensions. La longueur de la plupart des spécimens varie entre 1 et $3 \mathrm{~mm}$. Mais nous en rencontrons quelques uns qui atteignent $5 \mathrm{~mm}$. et dont le diamètre est d'environ $1 / 2 \mathrm{~mm}$ (Barrande et al., 1852).

Descrição: concha com tamanho aproximado de $1,8 \mathrm{~cm}$, reta, lisa e sem a presença de anelações. Em alguns casos, ocorre a presença de tênues estrias de crescimento. Câmara embrionária com forma goticular.

Distribuição geográfica: A) rio Maecuru, margem direita, $400 \mathrm{~m}$ à montante da cachoeira Teuapixuna ou Alagação, coletadas pela Expedição Orville A. Derby, 1986; B) afloramento localizado no lado norte de uma curva da BR-316 (Picos - Teresina), a cerca de $5 \mathrm{~km}$ do trevo de Picos em direção a Oeiras, dentro de uma propriedade particular, no município de Picos (Deusana Machado, comunicação pessoal, 2014).

Distribuição estratigráfica: Formação Maecuru (Eifeliano) e Formação Cabeças (Givetiano).

\section{DISCUSSÃO}

Os tentaculitoideos possuem uma ampla distribuição geográfica, sendo encontrados em locais como Afeganistão, África do Sul, Alemanha, Áustria, Argentina, Bolívia, Canadá, China, Estados Unidos, Paraguai, Peru, Polônia, República Tcheca e Suécia (Knod, 1908; Fisher \& Young, 1955; Ljashenko, 1955, 1957, 1959; Fisher, 1962; Bouček, 1964; Lardeux, 1969; Larsson, 1979; Lindemann \& Yochelson, 1992; Alberti, 1993; Lindemann \& Melycher, 1997; Azevedo-Soares, 1999; Wittmer \& Miller, 2011; Schindler, 2012; Wei et al., 2012), e apresentando distribuição estratigráfica do Ordoviciano ao Devoniano, possuindo ápice da classe no Devoniano Médio e extinção total da classe no limite Frasniano-Fameniano (Bond, 2006; Wittmer \& Miller, 2011; Schindler, 2012).

No Hemisfério Norte, uma grande quantidade de gêneros e espécies é conhecida, enquanto no Brasil são poucos os táxons encontrados no registro estratigráfico (Ciguel, 1989; Azevedo-Soares, 1999; Comniskey \& Ghilardi, 2013).

Os trabalhos referentes ao surgimento, apogeu e extinção dos tentaculitoideos são controversos. Autores como Wittmer \& Miller (2011) e Schindler (2012) citam a ocorrência dos primeiros tentaculitoideos no início do Ordoviciano. Porém ocorre uma lacuna no registro de tentaculitoideos durante o Ordoviciano Médio, que voltam a aparecer no final do Ordoviciano. Segundo 

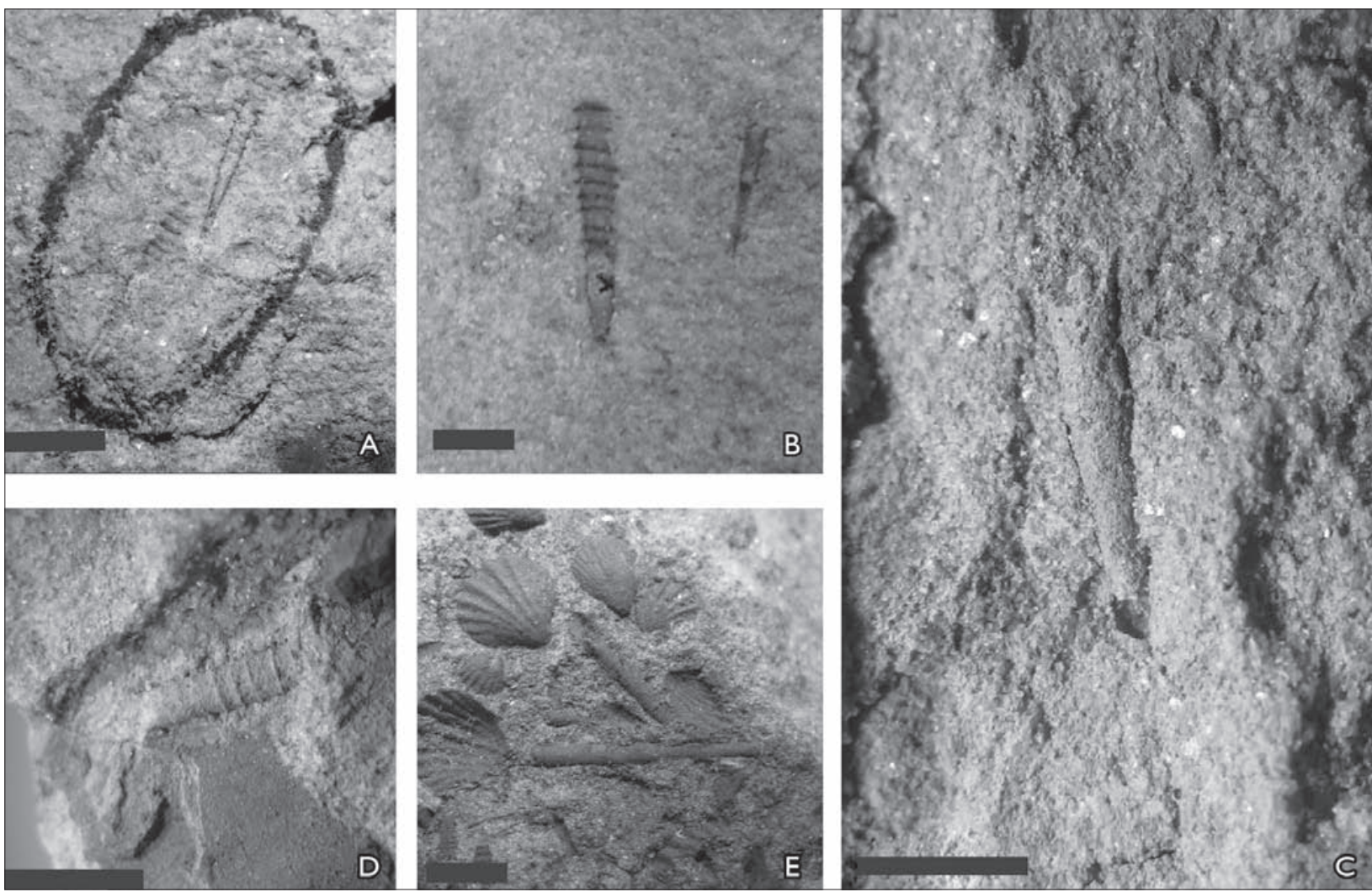

Figura 3. Tentaculitoideos das bacias do Amazonas e do Parnaíba: A) Tentaculites trombetensis (MN 2697), nota-se que os interespaços são mais largos e fundos do que o normal, sendo a característica principal desta espécie; B) Tentaculites stubeli (UNIRIO 5), os anéis oblíquos são características-chave nesta espécie; C) Styliolina clavulus (UNIRIO 42), a falta de ornamentação na concha caracteriza este gênero; D) Tentaculites eldredgianus (UNIRIO 41), os interespaços são maiores na região da abertura, como mostra a figura; E) Tentaculites oseryi (UNIRIO 58), anéis e interespaços com o mesmo tamanho são característicos nesta espécie. Escala $=0,5 \mathrm{~cm}$.

Wittmer \& Miller (2011), no início do Ordoviciano, o registro ocorre na Austrália, Malásia e Tailândia e, curiosamente, após esse período os tentaculitoideos não são documentados até o final do Ordoviciano. Neste período, muitas são as ocorrências da ordem Tentaculitida, existindo, no entanto, apenas um relato da ocorrência de Styliolina, marcando a primeira aparição dos Dacryoconarida.

O início do Siluriano é marcado por um notável aumento na diversidade de tentaculitoideos em todo o mundo (Fisher, 1962; Wittmer \& Miller, 2011; Wei et al., 2012).

Durante o Devoniano, o grupo mostra extensa disseminação global (Fisher, 1962; Alberti, 1993; Wittmer \& Miller, 2011; Wei et al., 2012; Schindler, 2012). Segundo
Wittmer \& Miller (2011), no início do Devoniano as três ordens estavam presentes em proporções praticamente iguais. Os Dacryoconarida atingem o máximo de sua diversidade durante o Devoniano Médio (Schindler, 2012), enquanto os Homoctenida têm seu ápice no Frasniano (Bond, 2006; Schindler, 2012).

Entre os trabalhos que relatam a distribuição geográfica dos tentaculitoideos durante o Ordoviciano, Siluriano e Devoniano, apenas o de Wittmer \& Miller (2011) registra sua presença na América do Sul, com ocorrências somente a partir do Devoniano, porém sem maiores detalhes quanto à localização das ocorrências e andares estratigráficos. No entanto, trabalhos de Clarke (1899b) já citam a ocorrência de tentaculitoideos para o Siluriano da

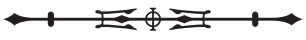


Bacia do Amazonas. Wei et al. (2012) afirmam ainda que não existem evidências convincentes quanto à presença de tentaculitoideos além dos trópicos.

O hábito de vida dos tentaculitoideos é assunto controverso. Fisher (1962) afirma que os tentaculitoideos eram animais tolerantes a diversos tipos de ambientes. Hoje em dia, sabe-se que os tentaculitoideos possuíam sua fase larval planctônica.

Nota-se que, no mesmo período (Devoniano), a diversidade de espécies da América do Sul é inferior a da Europa e da América do Norte. Como citado anteriormente, alguns autores acreditam que o grupo dos tentaculitoideos tivesse preferência por ambientes tropicais. Analisando os mapas de reconstrução paleogeográfica apresentado por Wittmer \& Miller (2011), verifica-se maior concentração dos registros do grupo em regiões mais tropicais, tais como Laurentia, Báltica e Avalonia, desde o Ordoviciano até o Devoniano.

Na América do Sul, os tentaculitoideos mais antigos que se têm registro são do Siluriano, com ocorrências no Brasil, Bolívia, Paraguai e Peru (Clarke, 1899b; Isaacson et al., 1976; Grahn, 1992; Ciguel, 1989; Heredia et al., 2007; Malanca et al., 2010). Até o momento, só existe registro do gênero Tentaculites para as camadas silurianas brasileiras.

Na América do Sul, durante o Devoniano, os tentaculitoideos ocorriam na Argentina, Bolívia, Brasil, Peru e Uruguai (Hartt \& Rathbun, 1875; Ulrich, 1893; Derby, 1890; Von Ammon, 1893; Katzer, 1897; Kayser, 1897, 1900; Siemiradzki, 1898; Clarke, 1899a; Schuchert, 1906;
Knod, 1908; Katzer, 1903, 1933; Clarke, 1913; Boucot et al., 1980). O registro pioneiro quanto ao surgimento dos primeiros dacryoconarida ocorre com o trabalho de Hartt (1897), com o gênero Styliolina. Ciguel \& Rosler (1985) fazem a primeira citação da ocorrência de Homoctenus e Volynites para as camadas do Devoniano da Bacia do Paraná.

Apesar do grande número de registro de tentaculitoideos para o Devoniano da América do Sul, verificou-se a necessidade de um refinamento sistemático, pois a citação da ocorrência, na maioria das vezes, não vem acompanhada por figuras ou fotos dos exemplares citados. Se o material apresenta-se mal preservado e sem classificação mais refinada, torna-se difícil obter estimativas quanto às prováveis rotas migratórias para o grupo.

Após as análises, foi verificada a ocorrência das espécies Tentaculites trombetensis, Tentaculites eldredgianus, Tentaculites stubeli, Tentaculites oseryi e Styliolina clavulus (Tabela 1) nas bacias do Amazonas e do Parnaíba. Na Tabela 1, é mostrada em qual formação cada espécie foi encontrada, assim como a idade atualizada das formações.

As quatro espécies de Tentaculites encontradas nas bacias do Amazonas e do Parnaíba foram citadas para o Devoniano da Bolívia e do Uruguai. Na Argentina, foi verificado o relato da ocorrência apenas da espécie T. crotalinus, e no Peru da espécie Tentaculites jaculus (sendo que esta espécie, no Brasil, só foi encontrada na Bacia do Paraná).

Clarke (1899a) e Katzer (1903) descrevem de forma pioneira a espécie Tentaculites crotalinus para as bacias do Amazonas e Parnaíba. Tal espécie tem seu holótipo descrito

Tabela 1. Distribuição das espécies de tentaculitoideos nas bacias do Amazonas (formações Maecuru e Ererê) e do Parnaíba (Formação Cabeças).

\begin{tabular}{c|c|c|c}
\hline \multirow{2}{*}{} & \multicolumn{2}{|c|}{ Bacia do Amazonas } & Bacia do Parnaíba \\
\cline { 2 - 4 } & $\begin{array}{c}\text { Formação Maecuru } \\
\text { (Eifeliano) }\end{array}$ & $\begin{array}{c}\text { Formação Ererê } \\
\text { (Neoeifeliano - Eogivetiano) }\end{array}$ & Formação Cabeças (Givetiano) \\
\hline Tentaculites eldredgianus & $\times$ & & $\times$ \\
\hline Tentaculites trombetensis & $x$ & $\times$ & $\times$ \\
\hline Tentaculites oseryi & & & $\times$ \\
\hline Tentaculites stubeli & $x$ & & \\
\hline Styliolina clavulus & & & $\times$ \\
\hline
\end{tabular}

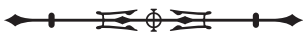


no Grupo Bokkeveld, na África (Salter, 1856). A presença desse táxon em camadas devonianas brasileiras indicaria uma provável comunicação remota entre essas bacias com o Grupo Bokkeveld. Após análises da diagnose original da espécie, observação de espécimes de T. crotalinus encontrados no Devoniano da Bacia do Paraná e examinar as figuras-tipo, constataram-se que os espécimes descritos por Katzer $(1897,1903)$ não se referem a T. crotalinus. As descrições e as figuras apresentadas nos trabalhos de Katzer (1897, 1903) não apresentam as mesmas características morfológicas, do espécime proposto por Salter (1856) como T. crotalinus. O mesmo fato ocorre com a espécie Tentaculites tenellus Katzer (1897), pois o autor apresenta uma diagnose pobre e figuras de baixa qualidade. $\bigcirc$ espécime não possui características morfológicas distintas o suficiente para a criação de uma nova espécie. Com base na descrição da espécie e na figura apresentada em seu trabalho, os presentes autores não reconheceram esta espécie em nenhuma das amostras analisadas. Schuchert (1906) apresenta uma tabela com os espécimes encontrados na Bacia do Amazonas, e nela o autor cita a ocorrência de $T$. tenellus, porém não apresenta uma descrição ou figura da espécie.

Um resultado importante foi que as cinco espécies encontradas nas bacias do Amazonas e do Parnaíba são diferentes daquelas encontradas na Bacia do Paraná. Verificou-se também que as espécies de tentaculitoideos encontrados na Bacia do Paraná ocorrem desde o Neopraguiano até o Neogivetiano, enquanto as espécies das bacias do Amazonas e do Parnaíba ocorrem em afloramentos datados do Eifeliano ao Givetiano. As espécies encontradas na Bacia do Paraná foram também localizadas em camadas do Devoniano da Bolívia. Esses dados nos levam a supor que, com base na ocorrência dessas espécies, não houve conexão e, portanto, migração entre as bacias intracratônicas brasileiras nessa época. Outra consideração importante seria o fato de que provavelmente esta fauna provenha da Bolívia, visto que a ocorrência do gênero Tentaculites tem seus primeiros registros durante o Siluriano da Bolívia, surgindo apenas no Neopraguiano da Bacia do Paraná e durante o Siluriano Inferior da Bacia do Amazonas.

Como citado em alguns trabalhos (Bond, 2006; Wittmer \& Miller, 2011; Schindler, 2012; Wei et al., 2012), a extinção dos tentaculitoideos ainda é motivo de discussões, sabendo-se que a extinção total do grupo ocorre no final do Devoniano. Bond (2006) afirma que os homoctenídeos apresentam seu ápice no Devoniano Médio e durante - limite Frasniano-Fameniano ocorre sua extinção, juntamente com a extinção total do grupo. Segundo Bond (2006), Wittmer \& Miller (2011) e Schindler (2012), a extinção do grupo ocorre concomitantemente a um evento de extinção conhecido como "crise de Kellwaser". Esta crise tem sido constantemente citada como a possível causa do desaparecimento dos tentaculitoideos do Hemisfério Norte, embora eles estivessem em competição com gastrópodes planctônicos, que estavam no auge de seu desenvolvimento. Ela é reconhecida como gradual, afetando primeiramente comunidades bentônicas por meio da anoxia. Eventuais pulsos anóxicos subsequentes reduziriam a extensão das áreas de ocorrência dos organismos pelágicos (Wittmer \& Miller, 2011).

Para Berkyová et al. (2007), o período Devoniano é considerado de grandes mudanças nas biosferas terrestre e marinha, o que resultou em perturbações no reino dos planctônicos (incluindo a extinção dos graptólitos, aparecimento dos dacryoconarídeos, declínio de acritarcas e extinção do tentaculitoideos no final do Devoniano).

Niko (2000) cita a ocorrência de homoctenídeos no limite Carbonífero/Permiano, porém a falta de dados sedimentológicos consistentes tornou esta ocorrência questionável (Schindler, 2012).

\section{CONCLUSÃO}

Das 39 amostras analisadas nas quatro instituições de ensino e pesquisa visitadas, foram reconhecidas as seguintes espécies de tentaculitoideos no Devoniano Médio da Bacia do Amazonas: Tentaculites trombetensis, T. stubeli, 
T. eldredgianus e S. clavulus. Na Bacia do Parnaíba, foram reconhecidas: T. trombetensis, T. oseryi, T. eldredgianus e $S$. clavulus. Diferentemente do que alguns autores haviam citado anteriormente, não foi reconhecida a espécie Tentaculites crotalinus nas bacias do Amazonas e do Parnaíba. Na América do Sul, o registro dos primeiros tentaculitoideos ocorreu durante o início do Siluriano, com o gênero Tentaculites. Representantes das ordens Dacryoconarida e Homoctenida foram encontrados a partir do Devoniano Inferior. Nas bacias do Amazonas e do Parnaíba só existe registro das ordens Tentaculitida e Dacryoconarida.

\section{AGRADECIMENTOS}

Comniskey agradece à Fundação de Amparo à Pesquisa do Estado de São Paulo (FAPESP) pela bolsa de doutorado concedida (2013/04884-0). Bosetti agradece ao Conselho Nacional Desenvolvimento Científico e Tecnológico (CNPq PQ 311483/2014-3). Os autores agradecem às instituições de ensino e pesquisa brasileiras que permitiram a análise do material estudado. Ao Prof. Dr. Antonio Carlos Sequeira Fernandes, pelas informações sobre o material de Clarke.

\section{REFERÊNCIAS}

ALBERTI, G. K. B., 1993. Dacryoconariden und homocteniden Tentaculiten des Unter-und Mittel-Devons I. Courier Forschungsinstitut Senckenberg 158: 1-230.

AZEVEDO-SOARES, H. L. C., 1999. Classe Tentaculita na Formação Ponta Grossa: uma proposta de sistematização: 1-117. Dissertação (Mestrado em Análise de Bacias e Faixas Móveis) Universidade do Estado do Rio de Janeiro, Rio de Janeiro.

BARRANDE, J., F. POČTA, J. PERNER, W. H. WAAGEN \& J. JAHN, 1852. Systéme Silurien du centre de la Bohéme. Chez l'Auteur, Prague.

BERKYOVÁ, S., J. FRYDA \& P. LUKES, 2007. Unsuccessful predation on Middle Paleozoic plankton: shell injury and anomalies in Devonian dacryoconarid tentaculites. Acta Palaeontologica Polonica 52(2): 407.

BLIND, W., 1969. Die systematische Stellung der Tentakuliten. Palaeontographica Abteilung A 133: 101-145.

BOND, D., 2006. The fate of the homoctenids (Tentaculitoidea) during the Frasnian-Famennian mass extinction (Late Devonian). Geobiology 4(3): 167-177.
BOUČEK, B., 1964. The Tentaculites of Bohemia: their morphology, taxonomy, ecology, phylogeny and biostratigraphy: 1-215. Publishing House of the Czechoslovak Academy of Sciences, Prague.

BOUCOT, A. J., P. E. ISAACSON \& G. LAUBACHER, 1980. An early Devonian, eastern Americas realm faunule from the coast of southern Peru. Journal of Paleontology 54(2): 359-365.

CIGUEL, J. H. G, 1989. Bioestratigrafia dos Tentaculitoidea no flanco oriental da Bacia do Paraná e sua ocorrência na América do Sul: 2 v. Dissertação (Mestrado em Geociências) - Universidade de São Paulo, São Paulo.

CIGUEL, J. H. G., O. ROSLER \& D. CAMINEIRO, 1984. Tentaculitoidea da formação Ponta Grossa no estado do Paraná. Anais do Congresso Brasileiro de Geologia 33: 655-669.

CIGUEL, J. H. G. \& O. ROSLER, 1985. Caracterização das formas brasileiras de Tentaculites crotalinus Salter, 1856. Anais da Academia Brasileira de Ciências 57(1): 116.

CIGUEL, J. H. G., O. ROSLER \& R. M. HOFMEISTER, 1987. Preservação parcial da concha de Tentaculites crotalinus, da Formação Ponta Grossa (Devoniano). Boletim IG-USP. Série Científica 18: 17-26.

CLARKE, J. M., 1899a. Moluscos devonianos do Estado do Pará, Brasil. Archivos do Museu Nacional 10(3-8): 49-174.

CLARKE, J. M., 1899b. A fauna siluriana superior do Rio Trombetas. Archivos do Museu Nacional 10: 1-48

CLARKE, J. M., 1913. Fósseis Devonianos do Paraná: 1-353. Monographias do Serviço Geológico e Mineralógico do Brasil, Rio de Janeiro.

COMNISKEY, J. C. \& R. P. GHILARDI, 2013. Levantamento histórico da Classe Tentaculitoidea no Devoniano brasileiro: principais características e padrões de preservação. Terr@ Plural 7(2): 115-126.

DERBY, O. A., 1890. Nota sobre a geologia e paleontologia de Matto Grosso. Imprensa Nacional, Rio de Janeiro.

FARSAN, N. M., 1994. Tentaculiten: Ontogenese, Systematik, Phylogenese, Bioestratonomie und Morphologie. Abhandlungen Der Senckenbergischen Naturforschenden Gesellschaft 547(1): 128.

FISHER, D. W., 1962. Small conoidal shells of uncertain affinities. In: R. C. MOORE (Ed.): Treatise on invertebrate paleontology: 98-143. Geological Society of America/University of Kansas Press, Boulder/Lawrence.

FISHER, D. W. \& R. S. YOUNG, 1955. The oldest known tentaculitid: from the Chepultepec Limestone (Canadian) of Virginia. Journal of Paleontology 29(5): 871-875.

FONSECA, V. M. M., 2004. Chonetoidea (Brachiopoda) do Devoniano Médio das Bacias do Amazonas e Parnaíba, Brasil. Arquivos do Museu Nacional 62(2): 193-215. 
GRAHN, Y., 1992. Revision of Silurian and Devonian strata of Brazil. Palynology 16(1): 35-61.

HARTT, C. F., 1897. A fauna devonica do rio Maecuru. Boletim do Museu Paraense de História Natural e Ethnographia 204-246.

HARTT, C. F. \& R. RATHBUN, 1875. Morgan expedition, 18701871: on the Devonian trilobites and mollusks of Ererê, Province of Para, Brazil. Annals of the Lyceum of Natural History of New York 11(13): 110-127.

HEREDIA, S., A. MESTRE \& J. P. MILANA, 2007. Reappraisal of the Silurian stratigraphy at Cerro del Fuerte section (San Juan, Argentina). Anais do Congresso de Paleontología y Bioestratigrafía de Latinoamérica 4: 195-200.

ISAACSON, P. E., B. ANTELO \& A. J. BOUCOT, 1976. Implications of a Llandovery (early Silurian) brachiopod fauna from Salta Province, Argentina. Journal of Paleontology 50(6): 1103-1112.

KATZER, F., 1897. Das Amazonas-Devon und seine Beziehungen zu den anderen Devongebieten der Erde. Sitzungsberichte der Königlichen B'hmischen Gesellschaft der Wissenschaften, Mathematisch-Naturwissenschaftliche Classe 46: 1-50.

KATZER, F., 1903. Grundzuge der Geologie des unteren Amazonasgebietes (dês Staates Pará in Brasilien): 1-298. Max Weg, Leipzig.

KATZER, F., 1933. Geologia do Pará (Brasil). Boletim do Museu Paraense Emílio Goeldi 9: 270.

KAYSER, E., 1897. Beiträge zur Kenntniss einiger paläozoischer Faunen Sud-Amerikas. Zeitschrift der Deutschen Geologischen Gesellschaft 49: 274-311.

KAYSER, E., 1900. Alguns fósseis paleozóicos do Estado do Paraná. Revista do Museo Paulista 4: 301-311.

KNOD, R., 1908. Devonische faunen boliviens. Neues Jahrbuch für Geologie und Paläontologie Beilage 25: 493-601.

LARDEUX, H., 1969. Nouvelles données sur la morphologie des Tentaculitoidea. Éditions du Centre National de la Recherche Scientifique 258(24): 5939-5942.

LARSSON, K., 1979. Silurian tentaculitids from Gotland and Scania. Fossil and Strata 11: 1-180.

LINDEMANN, R. H. \& E. L. YOCHELSON, 1992. Viriatellina (Dacryoconarida) from the Middle Devonian Ludlowville Formation at Alden, New York. Journal of Paleontology 66(2): 193-199.

LINDEMANN, R. H. \& D. A. MELYCHER, 1997. Tentaculites (Tentaculitoidea) from the Manlius Limestone (Lower Devonian) at Schoharie, New York. Journal of Paleontology 71(3): 360-368.
LJASHENKO, G. P., 1955. Novye dannye o sistimatike tentakulitov, novakiyi I stiliolii. Biulleten Moskovskogo Obschestva Ispytatelei Prirody, Otdel Geologichskii, Novaia Seriia 30: 94-95.

LJASHENKO, G. P., 1957. Systematics of the tentaculitoids, nowakiids, and styliolinids. Ezhegodnik Vsesoyuznogo Paleontologicheskogo Obshchestva 16: 82-99.

LJASHENKO, G. P., 1959. Devonian Conoconchia of the central and eastern parts of the Russian Platform:1-220. The All-Russian Research Institute of Oil, Moscow.

MALANCA, S., M. J. ARIS, M. A. BOSO, E. GALLARDO, E. M. BRANDÁN \& J. C. FERNÁNDEZ, 2010. Invertebrados fósiles de la formación Lipeón (Silúrico superior) del área Bermejo-los Toldos, noroeste argentino. Revista de La Asociacíon Geológica Argentina 66(1): 171-177.

MELO, J. H. G., 1985. A Província Malvinocáfrica no Devoniano do Brasil. Dissertação (Mestrado em Geociências) - Universidade Federal do Rio de Janeiro, Rio de Janeiro.

NIKO, S., 2000. Youngest record of tentaculitoids: Hidagaienites new genus from near the Carboniferous-Permian boundary in central Japan. Journal of Paleontology 74(3): 381-385.

PONCIANO, L. C. M. O., 2011. Fósseis devonianos da Bacia do Amazonas: Bookess, Florianópolis 1-143.

PONCIANO, L. C. M. D. O. \& D. M. D. C. MACHADO, 2007. Hábitos de vida da associação "Schuchertella" agassizi - Pthychopteria eschwegei, formação Maecuru, Devoniano, Bacia do Amazonas, Brasil. Anuário do Instituto de Geociências 30(1): 135-144.

PONCIANO, L. C. M. D. O., A. R. D. S. F. D. CASTRO, V. M. M. D. FONSECA \& D. M. D. C. MACHADO, 2012. Tafocenoses da Formação Pimenteira, devoniano da Bacia do Parnaíba, Piauí: mapeamento, inventário e relevância patrimonial. Anuário do Instituto de Geociências 35(1): 5-27.

SALTER, J. W., 1856. Description of Palaeozoic Crustacea and Radiata from South Africa. Geological Society of London Transactions 7(2): 215-224.

SCHEFFLER, S. M., 2010. Crinoides e Blastoides do Devoniano brasileiro. Tese (Doutorado em Geologia, - Universidade Federal do Rio de Janeiro, Rio de Janeiro.

SCHINDLER, E., 1990. The late Frasnian (Upper Devonian) Kellwasser Crisis. Lecture Notes in Earth Sciences 30: 151-159.

SCHINDLER, E., 2012. Tentaculitoids - an enigmatic group of palaeozoic fossils. In: J. A. TALENT (Ed.): Earth and life: global biodiversity, extinction intervals and biogeographic perturbations through time: 479-490. Springer, Netherlands.

SCHUCHERT, C., 1906. Geology of the Lower Amazon region. The Journal of Geology 14(8): 722-746. 
SIEMIRADZKI, J., 1898. Geologische reisebeobachtungen in Südbrasilien. Aus Der Kaiserlich-Königlichen Hof-Und Staatsdruckerei 107(1): 23-40.

ULRICH, A., 1893. Palaeozoische Versteinerungen aus Bolivien. Neues Jahrbuch, Beil. Band 8: 60-64.

VINN, O., 2010. Adaptive strategies in the evolution of encrusting tentaculitoid tubeworms. Palaeogeography, Palaeoclimatology, Palaeoecology 292(1-2): 211-221.

VINN, O., 2013. Cornulitid tubeworms from the Ordovician of eastern Baltic. Carnets de Geologie [Notebooks on Geology] 2013(3): 131-138.

VINN, O. \& P. D. TAYLOR, 2007. Microconchid tubeworms from the Jurassic of England and France. Acta Palaeontologica Polonica 52(2): 391-399.

VINN, O. \& M. ZATON', 2012. Phenetic phylogenetics of tentaculitoids - extinct, problematic calcareous tube-forming organisms. Geologiska Föreningen i Stockholm Förhandlingar (GFF) 134(2): 145-156.
VON AMMON, L., 1893. Devonische Versteinerungen von Lagoinha in Matto Grosso (Brasilien). Zeitschrift der Gesellschaft für Erdkunde zu Berlin 28: 352-366.

WEEDON, M. J., 1991. Microstructure and affinity of the enigmatic Devonian tubular fossil Trypanopora. Lethaia 24(2): 227-234.

WEI, F., Y. GONG \& H. YANG, 2012. Biogeography, ecology and extinction of Silurian and Devonian tentaculitoids. Palaeogeography, Palaeoclimatology, Palaeoecology 358-360: 40-50.

WITTMER, J. M. \& A. MILER, 2011. Dissecting the global diversity trajectory of an enigmatic group: the paleogeographic history of tentaculitoids. Paleogeography, Paleoeclimatology, Paleoecology 312(1-2): 54-65.

ZATON', M. \& P. D. TAYLOR, 2009. Microconchids (Tentaculita) from the Middle Jurassic of Poland. Bulletin of Geosciences 84(4): 653-660.

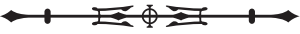


